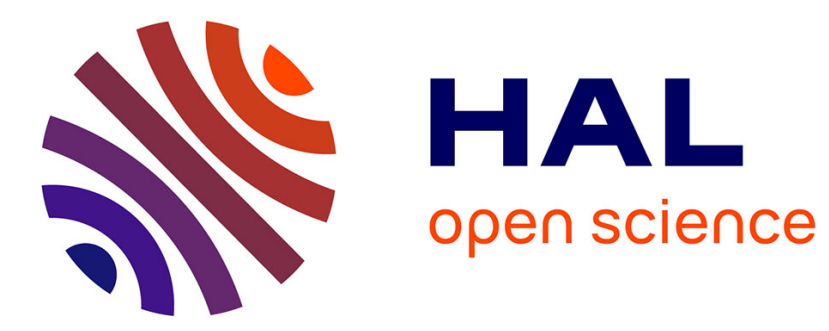

\title{
Photothermal Plasmonic Actuation of Micromechanical Cantilever Beams
}

\author{
M D Pichois, M A Hurier, M Vomir, A Barsella, B Donnio, J L Gallani, M V
} Rastei

\section{> To cite this version:}

M D Pichois, M A Hurier, M Vomir, A Barsella, B Donnio, et al.. Photothermal Plasmonic Actuation of Micromechanical Cantilever Beams. Physical Review Applied, 2021. hal-03281606

\section{HAL Id: hal-03281606 https://hal.science/hal-03281606}

Submitted on 8 Jul 2021

HAL is a multi-disciplinary open access archive for the deposit and dissemination of scientific research documents, whether they are published or not. The documents may come from teaching and research institutions in France or abroad, or from public or private research centers.
L'archive ouverte pluridisciplinaire HAL, est destinée au dépôt et à la diffusion de documents scientifiques de niveau recherche, publiés ou non, émanant des établissements d'enseignement et de recherche français ou étrangers, des laboratoires publics ou privés. 


\title{
Photothermal Plasmonic Actuation of Micromechanical Cantilever Beams
}

\author{
M. D. Pichois,${ }^{1}$ M. A. Hurier, ${ }^{1}$ M. Vomir, ${ }^{1}$ A. Barsella, ${ }^{1}$ B. Donnio, ${ }^{1}$ J. L. Gallani,${ }^{1}$ and M. V. Rastei ${ }^{* 1}$ \\ ${ }^{1}$ Institut de Physique et Chimie des Matériaux de Strasbourg, CNRS, \\ Université de Strasbourg, 23 rue du Loess, F-67034 Strasbourg, France
}

(Dated: February 16, 2021)

\begin{abstract}
Oscillations of charge carriers in plasmonic metal nanoparticles activated by resonant absorption of light are accompanied by local temperature increase due to non-radiative plasma damping. The control of this photothermal effect is considered essential for many applications ranging from photochemistry or nanomedicine to chemical/physical sensing. Here, we present a study on the conversion of visible light into mechanical energy via photothermal plasmonic and non-plasmonic effects. Using atomic force microscopy cantilevered sensors coated with various materials and excited in vacuum by a wavelength tunable laser, it is shown that light generates a resonant oscillation of the cantilever. The photoinduced oscillation amplitude depends on the wavelength of the incident light, allowing for an optimization of energy conversion based on absorption spectroscopy of the coating material. The effect of non-photonic forces acting on the cantilever is probed in the context of photoinduced force microscopy by placing the cantilever in interaction with a substrate surface at various distances. The findings are relevant for any technique utilizing an optical actuation of a mechanical system, and for photoinduced force detection methods in particular.
\end{abstract}

PACS numbers: 78.20.Nv, 82.80.Kq, 43.58.Wc, 07.79.Lh

\section{INTRODUCTION}

The photothermal effect relies on the partial conversion of optical energy into heat upon absorption of light by a medium $[1,2]$. The transferred energy may generate sustainable mechanical deformations [3, 4], enabling innovative far field spectroscopic applications [5-8]. For mechanical resonators with well-defined vibrational eigenmodes [9, 10], an intensity-modulated light induces periodic shape deformations $[11,12]$, and accumulations of energy into oscillations [13-15]. At resonance, a weakly damped mechanical oscillator can improve several fold the photothermal excitation efficiency because of the high oscillation quality factor [16-18]. This efficiency can furthermore be enhanced by using coatings presenting different thermoelastic coefficients [16, 19-21]. It is also possible to remove energy from a mechanical resonator through photothermal effects, allowing for active feedback cooling [22-26], and synchronization [27, 28].

Many of these photoinduced phenomena have been investigated in relation with photothermal deflection spectroscopy [6, 29, 30], a technique mostly developed for molecular spectroscopy in the infrared spectral range $[31,32]$, which in some cases makes use of miniature cantilever sensors [33-36]. The concept of actuation is there analogous to what is used in some advanced atomic force microscopes, where cantilever sensors are driven by modulated light [37-40]. This replaces the piezoelectric actuation, thus limiting the broadening effects and noise from spurious acoustic vibrations [38, 41]. Such a parametric photothermal excitation of AFM probes is very promising for scanning probe imaging in general, since it can greatly enhance the force sensitivity by also circumventing electrical cross-talks. However, the mechanical response of coated mechanical resonators, such as cantilever beams, when driven by optical excitation at various wavelengths across the visible spectral domain remains unexplored. This is critical for experiments conducted with tunable light sources, as needed for instance in microscopic photovoltage spectroscopy $[42,43]$, Kelvin probe force microscopy under illumination $[44,45]$, photo-piezoresponse and photoelectric force microcopy [46-48], or photoinduced force microscopy and spectroscopy [49-52].

Photoinduced force microscopy recently opened exciting opportunities for studying nanometer scale optical properties and light-matter interactions with unprecedent spatial resolution [49-53]. The central idea in this technique is the generation of optical forces between the apex of an atomic force microscope tip and a sample surface upon absorption of light by the latter [54-56]. Non-local photoinduced interactions with the microscope force sensor, i.e. away of the tip apex, have been found to strongly interfere with the measured signal $[49,57]$, allowing a development of phase-sensitive heterodyne detection methodes $[58,59]$. The photothermal effects generated by light absorption on the tip body and on the cantilever, are consequently of a particular relevance for photoinduced force microscopy and spectroscopy, where they deserve a supplementary attention.

Here, we report on the light-induced dynamics of micromechanical cantilevers coated with materials which absorb light in the visible spectral range. Using a tunable laser source modulated in intensity at specific frequencies, it is shown that the oscillation amplitude of the cantilever retraces the absorption spectrum of the coating material, leading in the case of gold nanoparticle coatings to noticeable plasmon-enhanced mechanical excitations. A specific absorption peak ( $545 \mathrm{~nm})$, induced by intra-band collective plasmonic excitations of conduction electrons is revealed. By substituting the Au NPs with a continuous metallic $\mathrm{Au}$ film, it is shown that interband electronic transitions - not being part of plasma 
oscillations but occurring in NPs as well [60, 61] - already induce a specific absorption of light in the visible range. This permits a deeper understanding of the photoinduced oscillations as a function of wavelength. Furthermore, it is revealed that, although less pronounced, the resulting spectra line shape persists when the cantilever is subjected to additional non-optical forces, i.e. van der Waals (vdW) and electrostatic. To this end, we enabled controlled near-field interactions with a substrate surface, which unveiled useful details about the effect of non-photothermal forces during actuation. In addition, results obtained with a molecular coating consisting in perylene molecules are also presented, strengthening the dominant role played by photothermal effects.

\section{EXPERIMENTAL DETAILS}

Experiments have been performed with a modified atomic force microscope coupled with a supercontinuum laser source and a monochromator. The setup is sketched in Fig. 1 (a). The intensity of the laser beam was modulated by a programmable broadband electrooptical modulator. A photometer allows feedback control of the incident laser power which was kept constant (optical fluence $\sim 50 \mu \mathrm{J} / \mathrm{cm}^{2}$ ) when changing the wavelength. The beam was focused with a lens at the tip-sample junction within a spot of about $25 \mu \mathrm{m}$ diameter. This value is approximately three times larger than the size of the basal plane of the pyramidal tip $(\sim 8 \mu \mathrm{m})$ which is attached at the free end of the cantilever. The width of the cantilevers is $\sim 30 \mu \mathrm{m}$. In such a configuration, nonlocal light-cantilever interactions, i.e. on the body of the vibrating cantilever, are important. The AFM was operating in vacuum at pressures below $10^{-3}$ mbar in order to maximize the $Q$ factor of the cantilever.

Cantilevers made of silicon and presenting different types of coatings were employed. A first type of cantilever was covered on the back side (detector side) with a continuous $\mathrm{Al}$ film, and with one monolayer of $\mathrm{Au}$ nanoparticles (NPs) on the front side (excitation side). The diameter of the NPs was $8 \pm 2 \mathrm{~nm}$. The NPs covered both the tip and cantilever body. After deposition, the cantilever had a spring constant $k=40 \mathrm{~N} / \mathrm{m}$ and first resonant mode $f_{0}=305 \mathrm{kHz}$. The NPs were deposited by Langmuir-Blodgett technique which allows a rather good deposition homogeneity on the cantilever [Fig. 1(b)]. The Al back-coating increases the sensitivity due to the difference of thermal expansion between the Si and Al. A second type was coated on the front and back side with $50 \mathrm{~nm}$ of a continuous Au film [Fig. 1(c)]. After deposition $k$ was $21 \mathrm{~N} / \mathrm{m}$ and the fundamental resonant frequency $f_{0}=140 \mathrm{kHz}$. A third cantilever type consisted in a molecular film of perylene deposited on the front side of a cantilever coated with $\mathrm{Al}$ on the back side. The substrate surface was an indium tin oxide (ITO) film grown on glass. The electrical conductivity of ITO is not playing any particular role throughout this study. The
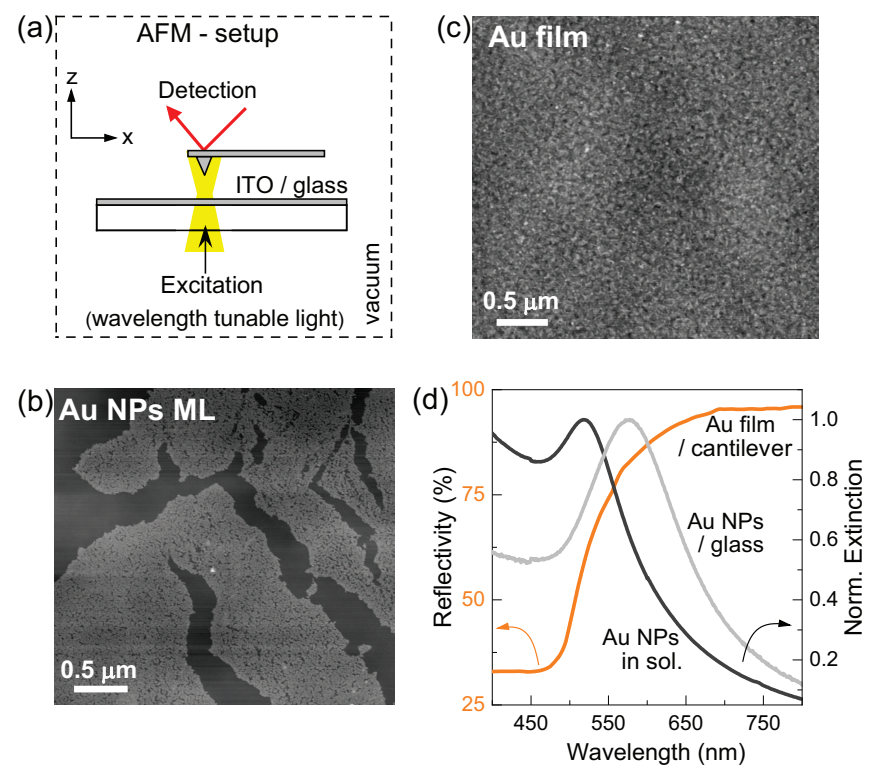

FIG. 1. (a) Schematic of the setup used for wavelengthdependent photothermal actuation experiments. The tipsubstrate surface distance is controlled by a z-piezo (not shown). (b) AFM image $\left(3 \times 3 \mu \mathrm{m}^{2}\right)$ of $1 \mathrm{ML}$ of $\mathrm{Au}$ NPs acquired on the front side of a cantilever. Image taken in a zone with cracks, allowing a better visualization of the layer (ML height $=10 \pm 2 \mathrm{~nm}) . \quad(\mathrm{c})$ AFM image $\left(3 \times 3 \mu \mathrm{m}^{2}\right)$ of the $\mathrm{Au}$ film (roughness $<10 \mathrm{~nm}$ ) recorded on the front side of a cantilever. (d) Orange line corresponds to the reflectivity of $\mathrm{Au}$ film measured on the cantilever and part of its support. Grey lines are normalized optical extinction of $\mathrm{Au}$ NPs (1 ML) deposited on a glass substrate (light gray), and dispersed in a toluene solution (dark gray). Spectra obtained by macroscopic optical spectrometry.

oscillation amplitude of the cantilever was continuously measured with two lock-in amplifiers, tuned to the first two flexural resonant modes.

\section{RESULTS AND DISCUSSION}

For an overview of the optical properties of the coated probes, we first performed standard optical spectroscopy characterizations using a UV-Vis spectrometer. The probes coated with a Au film were measured by reflectivity, while illuminating the whole cantilever, the tip, and about one third of the cantilever holder [orange curve in Fig. 1(d)]. As expected, the Au film spectrum presents a progressive increase of reflectivity when the wavelength is changed from near-UV spectral range to about 675 $\mathrm{nm}$. This increase is the result of a progressive decrease of $\Delta X$ and $\Delta L$ inter-band transitions from the occupied $s p$ - to unoccupied $d$-bands, which is a known wavevectordependent absorption effect in $\mathrm{Au}$ [62].

The reflectivity signal probing the Au NPs deposited on the cantilever was found too weak to reliably inform about light absorption. Hence, using the same Langmuir- 


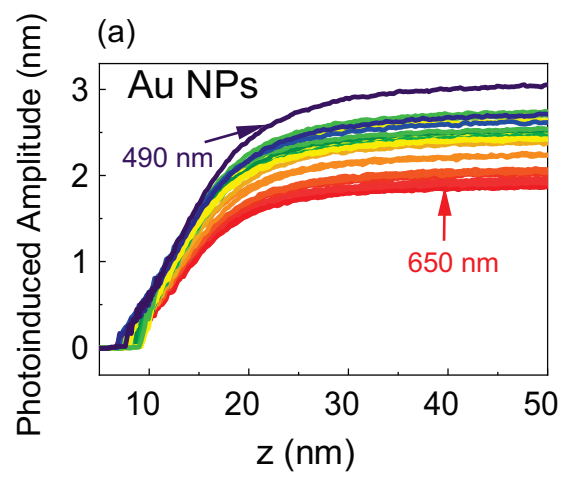

(b)

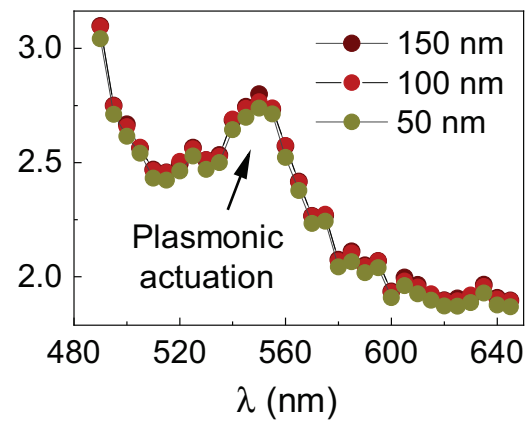

(c)

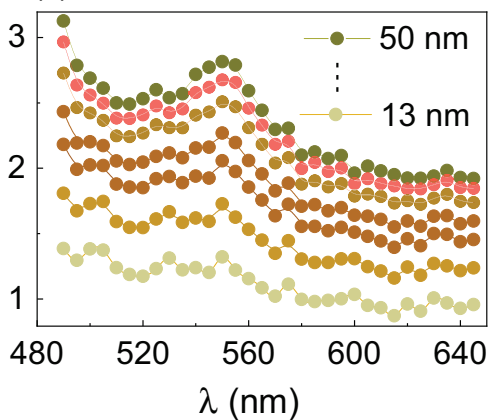

FIG. 2. Photoinduced supra-resonance actuation of a cantilever coated on the front side with 1 ML of Au NPs. Back-side coating: continuous Al film of $45 \mathrm{~nm}$ thickness. (a) Photoinduced oscillation amplitude while approaching the tip to the surface at different excitation wavelengths. (b) and (c) Wavelength-dependent photoinduced amplitude signal at few fixed tip-sample distances, i.e. z-values, extracted from (a). The number of data points is equal to the number of the approach-retract curves. Each color indicates a value of $\mathrm{z}$, as labeled. The other curves in (c) are for $\mathrm{z}=29,23,19,17,15 \mathrm{~nm}$, respectively.

Blodgett procedure as for NPs deposition on the cantilever, we deposited one ML of Au NPs on a transparent glass substrate and performed optical extinction measurements in transmission [gray curves in Fig. 1 (d)]. An extinction spectrum of NPs dispersed in a solution is also shown in Fig. 1 (d) for comparison. This gives an idea about the impact of the substrate proximity on the absorption properties of the NPs [60] which, as seen, slightly red-shifts the peak position in the spectra. The peak, falling at $\sim 520 \mathrm{~nm}$ for NPs in solution and at $\sim 565 \mathrm{~nm}$ after the their deposition on glass, is given by the local plasmon resonance in NPs which enhances the absorption of light.

\section{A. Photothermal plasmonic actuation: intra-band transitions}

To efficiently detect the photothermal actuation, the light-intensity modulation frequency $\left(f_{m}\right)$ was chosen close to the first mechanical eigenmode of the cantilever $\left(f_{0}\right)$. Such a near-resonant excitation strongly increases the oscillation amplitude of the cantilever. The surface force gradients along $\mathrm{z}$-axis are able to change the resoannce frequency $f_{0}$ of the cantilever. Depending on the sign of the force (attractive or repulsive), $f_{0}$ can shift towards smaller or higher frequencies. Note that,with typical cantilevers having spring constants below $80 \mathrm{~N} / \mathrm{m}$, a dominant repulsive regime is nevertheless difficult to access by approaching the tip to a surface. This is firstly because $\mathrm{vdW}$ and electrostatic forces are inherently attractive in an AFM configuration, and secondly because the tip will jump to contact well before short-range repulsive forces are reached $[63,64]$. This stops the oscillatory behavior of the cantilever, as discussed below.

Figure 2 shows the photoinduced oscillation amplitude of a cantilever covered with $\mathrm{Au}$ NPs for various wavelengths when approaching the tip to the surface down to contact. The upper violet curve corresponds to the smaller wavelength used. It is first seen that the cantilever dynamics is characterized by a constant amplitude at large distances (see SM [65] for even larger $z$ values), and a progressive decrease of the signal when approaching the cantilever to the ITO substrate. Such a decrease is explained by the fact that the modulation frequency $f_{m}$ was set slightly larger (supra-resonance) than $f_{0}\left(f_{m}-f_{0}\right.$ $=\Delta f=5 \mathrm{~Hz}$ in the present case).

At small distances, vdW and electrostatic forces interact attractively with the tip, displacing $f_{0}$ towards smaller values. The jump-to-contact is evidenced by the fact that the photoinduced oscillation amplitude of the cantilever comes to zero, since the photothermal effects cannot any more efficiently excite the cantilever. This occurs in Fig. 2 (a) between 7 and $10 \mathrm{~nm}$ above the surface, as also confirmed by the mean deflection signal of the cantilever (not shown). This is furthermore confirmed by the oscillation amplitude of the slightly piezoelectrically excited $f_{1}$ bending mode (see SI).

By analyzing the photoinduced amplitude in Fig. 2(a) at fixed tip-surface distances, spectra at different $z$-values can be obtained. Figure 2(b) displays three spectra at three large tip-sample distances. These spectra are almost identical, presenting a very similar variation of the photoinduced signal with the wavelength. Importantly, in all spectra there is a peak at about $545 \mathrm{~nm}$ induced by the localized surface plasmon resonance of NPs. Moreover, the peak appears superimposed to an overall decrease of the signal with wavelength. This spectral line shape is reminiscent of the extinction signal observed for NPs in Fig. 1(d). In addition, the photoinduced spectra features are found to persist up to the jump-to-contact when approaching the surface [Fig. 2 (c)]. Whether in Fig. 2 (c) there are additional short-range tip-sample photoinduced forces - as used in photoinduced force microscopy for instance - remains an open question, as such forces are also expected to depend on the wavelength.

The photothermal excitation mechanism is therefore based on the wavelength-dependent optical absorption of 
the adsorbed NPs. The resulting local heating and heat transfer causes a periodic thermal expansion in the cantilever body at the modulation frequency $f_{m}$. The thermal expansion is not expected to be homogeneous across the cantilever thickness due to the heat propagation and dissipation effects. This mechanism can significantly be enhanced in the presence of bimorph effects induced by a back-side coating $[41,66]$.

Two conclusions can be driven at this stage from the above experiments. Firstly, the photoinduced oscillation amplitude of the cantilever finely retraces the wavelength-dependent absorption properties of the NPs, and secondly the ability to convert light into mechanical energy at the localized plasmon resonance via photothermal effect holds for both inter- and intra-band photoelectron transitions. The fact that these transitions are observed in photoinduced oscillation amplitude and that their relative strengths are as in macroscopic extinction spectra, speaks in favor of a similar photothermal efficiency resulting from inter-and intra-band transitions.

\section{B. Photothermal actuation and inter-band electronic transitions in gold}

In the case of cantilevers coated with a continuous Au film, intra-band photoelectron transitions are not expected to play a significant role in visible spectral range. This constitutes an interesting opportunity to verify the capability of inter-band transitions to actuate alone the cantilever. Experiments, as described above in the case of $\mathrm{Au} \mathrm{NPs,}$ were therefore also performed with cantilevers covered with continuous Au films. The photoinduced amplitude signals when bringing the tip to the surface are shown in Fig. 3 (a). The resulting $z$-dependent spectra reveal a different variation with wavelength as compared to the Au-NPs case. In particular, the spectra shape is a progressive decrease of signal with wavelength [Fig. 3 (b)]. This strongly recalls the effect of inter-band absorption deduced from reflectivity spectrum in Fig. 1(d) in the respective wavelength interval. The photoinduced signal is again persisting up to the jump to contact, event which is now explicitly shown as an example in Fig. 3 (c). The spectral signal starts in this case to decrease at about $15 \mathrm{~nm}$ from the surface, as seen in Fig. 3 (d). Indeed, it is observed that despite a significant overall reduction of the signal the overall shape of spectra is similar up to the jump-to-contact point. Therefore, a peak induced by plasmonic intra-band transitions, which is specific to NPs, is not any more observed. Nonetheless, it is interesting to observe that inter-band transitions also represent a source of actuation for cantilevers covered with $\mathrm{Au}$ continuous metallic films.
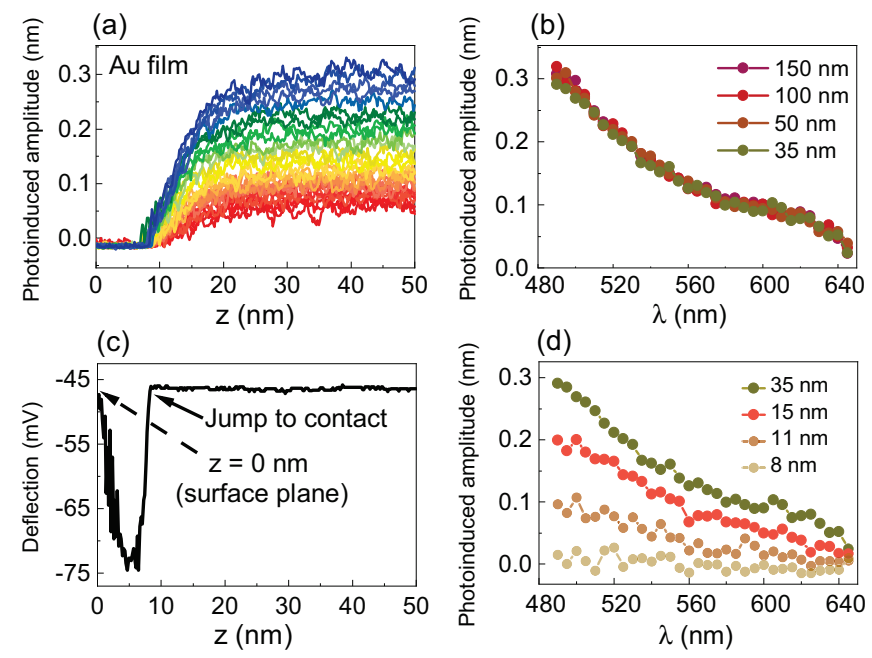

FIG. 3. Photoinduced supra-resonance actuation of a probe coated with a continuous Au film on both back- and front-side. (a) Photoinduced amplitude signal while approaching the tip to the surface, at different excitation wavelengths (different curves). Lower and upper curves are 645 and $490 \mathrm{~nm}$. (b) and (d) Wavelength-dependent photoinduced amplitude signal at several fixed tip-sample distances, i.e. z values. Curves obtained by using the data from (a), at several z-values, as labeled. Note the absence of signal upon tip-surface contact in the curve corresponding to $8 \mathrm{~nm}$. (c) Example of static deflection signal of the cantilever as a function of $\mathrm{z}$, showing the position of the jump to contact and of the surface plane.

\section{Supra/sub-resonant photothermal actuation}

The focalisation of the laser beam produces an inherent illumination of the upper part of the tip and of the cantilever end. At large tip-surface distances, the influence of non-optical surface forces can be neglected. In this case, the cantilever can be modeled as a harmonic oscillator under the influence of photoinduced effects. As the tip, which is attached at the free end of the cantilever, approaches the substrate surface, the cantilever dynamics is driven by a combination of photoinduced actuation and local surface forces $F_{\text {int }}(z)$ which are distance-dependent. The photoinduced actuation is correlated to the amount of light absorption via photothermal effects. Note that the photothermal effects are large in coated cantilevers due to the bimorph effect $[41,66]$. This makes negligible the contribution of other photoinduced driving mechanisms, such as photovoltage [67] or radiation pressure [68] effects. The resulting equation of motion including the photothermal actuation and the surface forces can then be written as:

$$
m_{\mathrm{eff}} \ddot{z}=-k z-\frac{\sqrt{k m_{\mathrm{eff}}}}{Q} \dot{z}+F_{\mathrm{PT}} \cos \left(2 \pi f_{m} t\right)+F_{\mathrm{int}}(z)
$$

where $m_{\text {eff }}$ is the effective mass of the oscillator, $k$ is the spring constant, $Q$ is the quality factor, and $F_{\mathrm{PT}} \cos \left(2 \pi f_{m} t\right)$ is the time-dependent photothermal ex- 
citation. The thermal noise and the eventual static deviations of the mean position of the cantilever due to inhomogeneous thermal expansion coefficients are also expected small, hence not included in the equation. As the photothermal driving is found independent of $z$ (at large z-values), this term should not have a strong spatial gradient in our optical system. In addition, although photothermal effects are expected to induce small periodic temperature variations, an overall change of the resonant frequency of the cantilever $f_{0}$, at large tip-surface distances, was not observed in our case. This is at variance with single nanoparticle spectroscopy cases where illumination powers and $\mathrm{Q}$ values are larger $[69,70]$. The average temperature variation due to the absorption of light is therefore small in our case, without a significant impact on $k$ and hence on $f_{0}$. Instead, when the tip approaches the substrate surface the $F_{\text {int }}(z)$ term, which has a $z$-dependence, can induce variations of $f_{0}$. This has a significant impact on the photothermal excitation efficiency.

The decrease of spectra intensity when approaching the contact can be explained through the dynamics of the $f_{0}$ mode [71], which is progressively shifted downward by vdW and electrostatic forces. With an initial $f_{m}$ set above $f_{0}$ (supra-resonance regime), a downward shift of $f_{0}$ translates in a less efficient photothermal excitation because of a gradual out-of-resonance actuation. It is worth noticing that, while photoinduced signal is in $\mathrm{mV}$ in Fig. 2, it is in volts in Fig. 3. This is because for the experiment shown in Fig. 2 the cantilever was coated with $\mathrm{Au}$ on the backside as well, whereas in that of Fig. 3 the cantilever had an Al coating on the backside. Such structural asymmetry, with materials having different thermal expansion coefficients results in amplifications of the photothermal-induced amplitudes.

The effect of setting $f_{m}$ in supra- or sub-resonance regime is shown in Fig. 4 for a cantilever covered with $\mathrm{Au}$ NPs. As seen, in both supra-resonance (red curves) and sub-resonance (green curves) actuation regimes, there is a strong dependence of photoinduced amplitude with the choice of $f_{m}$ with respect to $f_{0}$, i.e. sign and magnitude of $\Delta f$. As the photoinduced amplitude depends on $\Delta f$ and photothermal effect, it is important to analyze their relative impact on the cantilever amplitude.

A downward shift of $f_{0}$ mode induced by attractive surface forces manifests differently in supra-resonance and $s u b$-resonance actuation regimes. As in the first case $\Delta f>0$, the shift leads to an incremental difference between $f_{0}$ and $f_{m}$, progressively pushing the system out of the resonance. This translates in a continuous drop of the photoinduced amplitude (red curves in Fig. 4). The exact variation depends on the initial value of $\Delta f$. Conversely, in the sub-resonance regime $(\Delta f<0)$, the downward shift of $f_{0}$ mode implies that at some point $f_{m}=f_{0}$, which results in a maximum of the photoinduced oscillation amplitude [Fig. 4 (a)]. Afterwards, the photoinduced oscillation amplitude decreases due to the continuously downward shift of $f_{0}$ mode.
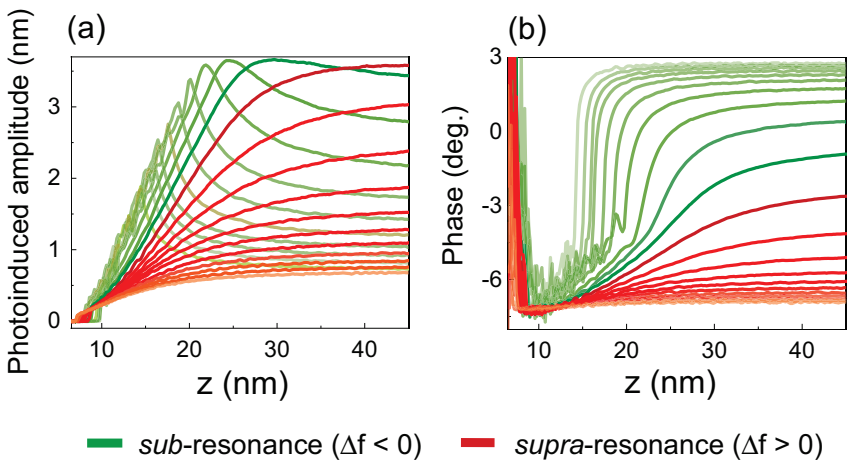

FIG. 4. Dependence of the photoinduced amplitude on the modulation frequency $f_{m}$. Distance dependence of the photoinduced amplitude (a) and of the phase (b) with $\Delta \mathrm{f}$ gradually tuned from $+50 \mathrm{~Hz}$ to $-50 \mathrm{~Hz}$. A cantilever coated with $\mathrm{Au}$ NPs was used (the same as in Fig. 1).

The passage from supra- to sub-resonance regime when changing the sign of $\Delta f$ was also found to impact the photoinduced oscillation phase [Fig. 4 (b)]. The variation of the phase shows that in $s u b$-resonance regime, it is possible to have a large region with a constant phase when decreasing the tip-surface distance (upper green curves), although such a plateau-like signal is followed by a sharp change of sign and a subsequent erratic dynamics at smaller distances. This distance interval is very interesting for detecting surface forces through phase detection. Note however that the phase is sensitive to the gradient of the force. There is also an inflection point, well distinguishable around $20 \mathrm{~nm}$ for few green curves. This inflection marks the fact that the phase sensitivity close to the resonance is very sharp. These inflection points observed in Fig. 4 (b) for the green curves are most likely induced by the vdW interactions which become stronger close to the surface than electrostatic interactions which dominate at larger distances.

All these different cantilever dynamics indicate that the photoinduced signals at a fixed wavelength have the origin in the photothermal actuation of the cantilever, which depends on $f_{0}$ mechanical mode shift when the tip experiences additional forces such as generated by a nearby surface. Any local photoinduced interaction between the tip apex and a sample surface, such as dipoledipole interactions considered so far in photoinduced force microscopy, must therefore be rationalized within this picture of an inherently non-local photothermal driving of the probe, regardless of the detection scheme.

\section{Absorption effects with a perylene coating}

In order to check for eventual effects introduced by electromagnetic field enhancements due to $\mathrm{Au}$, we also carried out experiments with a perylene molecular layer instead of $\mathrm{Au}$, in which case field enhancements are not expected. The solid line in Fig. 5 shows a macroscopic 


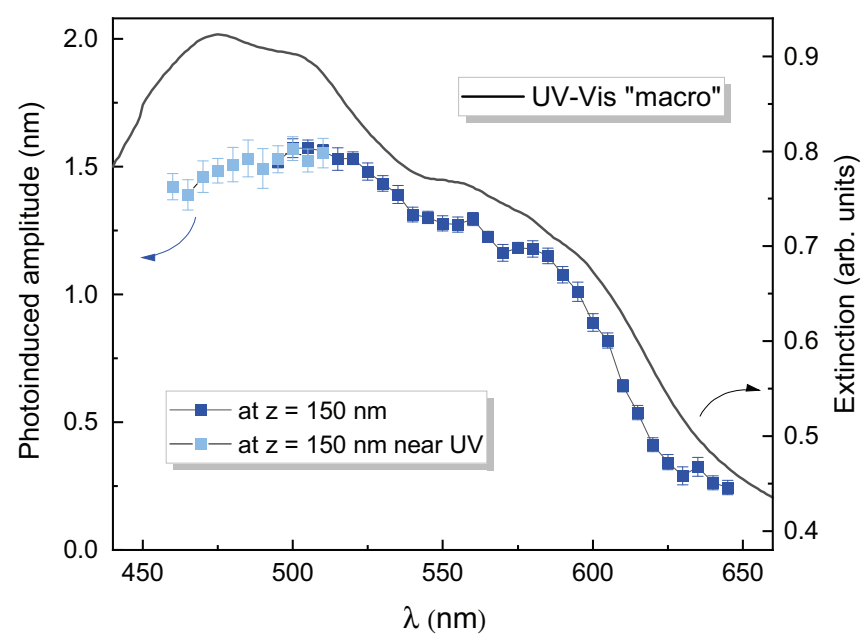

FIG. 5. Spectrum (blue data) of a cantilever covered with a perylene molecular film on the front-side, and a continous Al film on the back-side. Spectrum acquired at a tip-surface distance of $150 \mathrm{~nm}$. The near UV part of the spectrum (light blue) was obtained after a slight modification of the optical setup needed to access the respective wavelength interval. Solid line is a "macroscopic" extinction spectrum of a perylene film deposited on a glass substrate.

UV-Vis extinction spectrum acquired with a perylene film deposited on a glass substrate. The spectral features (various "shoulders") have their origin in the multiple absorption peaks known for perylene molecules in dilute solutions. As expected, the aggregation and formation of a solid-state film as well as the deposition on the substrate induced a broadening and a red shift of the respective peaks. More importantly, it is very interesting to see that the photoinduced amplitude signal traced for various wavelength (blue data) matches rather well the macroscopic extinction spectrum. Although the photothermal heat transfer mechanism from the molecular film to the cantilever must have differences with respect to the case of Au coatings, the results of Fig. 5 strongly support the fact that the photothermal effects are the main source of excitation of the cantilever dynamics.

\section{CONCLUSION}

In conclusion, this study reveals local and non-local photothermal actuation effects in the oscillatory dynamics of coated micromechanical cantilevers illuminated with light. The coatings of the cantilevers were found to play a critical role in the light induced mechanical dynamics. A light-induced actuation of cantilevers was found all across the visible spectral range. The conversion of photon energy into mechanical work via photothermal effects was nevertheless found particularly strong at wavelengths matching the localized surface plasmon resonances of $\mathrm{Au}$ NPs coatings. The variation of the photoinduced oscillation amplitude while modulating the intensity of the light close to an eigenmode of the cantilever, was moreover found to retrace the typical macroscopic absorption spectra of the coatings, highlighting the fact that the absorbed radiation energy is transferred into momentum. It was also shown that the choice of the light modulation frequency plays an important role, effect of a specific importance when additional non-optical forces are concomitantly applied to the cantilever. These findings indicate that the photothermal actuation governs the cantilever dynamics at large tip-sample distances, but interferes with the mechanical mode shift when the cantilever is, for instance, engaged below few tens $\mathrm{nm}$ to a substrate surface. This foretells a complex interplay between non-local photothermal effects and detection of photoinduced forces at surfaces.

We thank N. Beyer for technical assistance. This work was supported by the French National Research Agency (ANR) through contracts: ANR OH-RISQUE SMARAGD (14 OHRI 0008 01), EquipEx UNION No. ANR-10-EQPX-52, and ANR-17-EURE-0024.
[1] A. C. Tam, Applications of Photoacoustic Sensing Techniques, Rev. Mod. Phys. 58, 381 (1986).

[2] C. K. N. Patel and A. C. Tam, Pulsed Optoacoustic Spectroscopy of Condensed Matter, Rev. Mod. Phys. 53, 517 (1981).

[3] P. Hess, editor, Photoacoustic, Photothermal and Photochemical Processes at Surfaces and in Thin Films, Vol. 47 (Springer Berlin Heidelberg, Berlin, Heidelberg, 1989).

[4] L. C. Aamodt and J. C. Murphy, Thermal Effects in Photothermal Spectroscopy and Photothermal Imaging, J. Appl. Phys. 54, 581 (1983).

[5] J. C. Murphy and L. C. Aamodt, Photothermal Spectroscopy Using Optical Beam Probing: Mirage Effect, J. Appl. Phys. 51, 4580 (1980).

[6] W. B. Jackson, N. M. Amer, A. C. Boccara, and D.
Fournier, Photothermal Deflection Spectroscopy and Detection, Appl. Opt. 20, 1333 (1981).

[7] H. Vargas and L. C. M. Miranda, Photoacoustic and Related Photothermal Techniques, Physics Reports, 161, 43 (1988).

[8] S. E. Bialkowski, N. G. C. Astrath, and M. A. Proskurnin, Photothermal Spectroscopy Methods (Wiley, 2019).

[9] T. P. Weihs, S Hong, J. C. Bravman, and W. D. Nix, Mechanical Deflection of Cantilever Microbeams: A New Technique for Testing the Mechanical Properties of Thin Films, J. Mater. Res. 3, 931 (1988).

[10] B. Ilic, D. Czaplewski, M. Zalalutdinov, H. G. Craighead, P. Neuzil, C. Campagnolo, and C. Batt, Single Cell Detection with Micromechanical Oscillators, J. Vac. 
Sci. Technol. B Microelectron. Nanom. Struct. 19, 2825 (2001).

[11] M. Zhang, G. S. Wiederhecker, S. Manipatruni, A. Barnard, P. McEuen, and M. Lipson, Synchronization of Micromechanical Oscillators Using Light, Phys. Rev. Lett. 109, 233906 (2012).

[12] A. Mari and J. Eisert, Gently Modulating Optomechanical Systems, Phys. Rev. Lett. 103, 213603 (2009).

[13] T. J. White, N. V. Tabiryan, S. V. Serak, U. A. Hrozhyk, V. P. Tondiglia, H. Koerner, R. A. Vaia, and T. J. Bunning, A High Frequency Photodriven Polymer Oscillator, Soft Matter 4, 1796 (2008).

[14] K. D. Harris, R. Cuypers, P. Scheibe, C. L. Van Oosten, C. W. M. Bastiaansen, J. Lub, and D. J. Broer, Large Amplitude Light-Induced Motion in High Elastic Modulus Polymer Actuators, J. Mater. Chem. 15, 5043 (2005).

[15] T. Inoue, Y. Anno, Y. Imakita, K. Takei, T. Arie, and S. Akita, Optical Manipulation of Nonlinear Vibration of Graphene Mechanical Resonator, in Optical Manipulation Conference, edited by T. Omatsu, Vol. 10712 (SPIE, 2018), p. 59.

[16] D. Kiracofe, K. Kobayashi, A. Labuda, A. Raman, and H. Yamada, High Efficiency Laser Photothermal Excitation of Microcantilever Vibrations in Air and Liquids, Rev. Sci. Instrum. 82, 013702 (2011).

[17] B. Ilic, S. Krylov, K. Aubin, R. Reichenbach, and H. G. Craighead, Optical Excitation of Nanoelectromechanical Oscillators, Appl. Phys. Lett. 86, 1 (2005).

[18] N. V. Lavrik and P. G. Datskos, Femtogram Mass Detection Using Photothermally Actuated Nanomechanical Resonators, Appl. Phys. Lett. 82, 2697 (2003).

[19] O. Marti, A. Ruf, M. Hipp, H. Bielefeldt, J. Colchero, and J. Mlynek, Mechanical and Thermal Effects of Laser Irradiation on Force Microscope Cantilevers, Ultramicroscopy 42-44, 345 (1992).

[20] E. Merced, N. Dávila, D. Torres, R. Cabrera, F. E. Fernández, and N. Sepúlveda, Photothermal Actuation of V O2:Cr-Coated Microcantilevers in Air and Aqueous Media, Smart Mater. Struct. 21, 105009 (2012).

[21] N. Inada, H. Asakawa, T. Kobayashi, and T. Fukuma, Efficiency Improvement in the Cantilever Photothermal Excitation Method Using a Photothermal Conversion Layer, Beilstein J. Nanotechnol. 7, 409 (2016).

[22] I. Favero, C. Metzger, S. Camerer, D. König, H. Lorenz, J. P. Kotthaus, and K. Karrai, Optical Cooling of a Micromirror of Wavelength Size, Appl. Phys. Lett. 90, 104101 (2007).

[23] G. Jourdan, F. Comin, and J. Chevrier, Mechanical Mode Dependence of Bolometric Backaction in an Atomic Force Microscopy Microlever, Phys. Rev. Lett. 101, 133904 (2008).

[24] C. Metzger, M. Ludwig, C. Neuenhahn, A. Ortlieb, I. Favero, K. Karrai, and F. Marquardt, Self-Induced Oscillations in an Optomechanical System Driven by Bolometric Backaction, Phys. Rev. Lett. 101, 133903 (2008).

[25] S. De Liberato, N. Lambert, and F. Nori, Quantum Noise in Photothermal Cooling, Phys. Rev. A, 83, 033809 (2011).

[26] R. A. Barton, I. R. Storch, V. P. Adiga, R. Sakakibara, B. R. Cipriany, B. Ilic, S. P. Wang, P. Ong, P. L. McEuen, J. M. Parpia, and H. G. Craighead, Photothermal SelfOscillation and Laser Cooling of Graphene Optomechanical Systems, Nano Lett. 12, 4681 (2012).

[27] S. Houri, S. J. Cartamil-Bueno, M. Poot, P. G.
Steeneken, H. S. J. Van der Zant, and W. J. Venstra, Direct and Parametric Synchronization of a Graphene Self-Oscillator, Appl. Phys. Lett. 110, 073103 (2017).

[28] K. Shlomi, D. Yuvaraj, I. Baskin, O. Suchoi, R. Winik, and E. Buks, Synchronization in an Optomechanical Cavity, Phys. Rev. E, 91, 032910 (2015).

[29] A. C. Boccara, W. Jackson, N. M. Amer, and D. Fournier, Sensitive Photothermal Deflection Technique for Measuring Absorption in Optically Thin Media, Opt. Lett. 5, 377 (1980).

[30] W. B. Jackson and N. M. Amer, Direct Measurement of Gap-State Absorption in Hydrogenated Amorphous Silicon by Photothermal Deflection Spectroscopy, Phys. Rev. B 25, 5559 (1982).

[31] M. J. D. Low, C. Morterra, and A. G. Severdia, Infrared Study of Solid Cyclopentanol, Spectrosc. Lett. 15, 415 (1982).

[32] M. J. D. Low, C. Morterra, A. G. Severdia, and M. Lacroix, Infrared Photothermal Deflection Spectroscopy for the Study of Surfaces, Appl. Surf. Sci. 13, 429 (1982).

[33] J. R. Barnes, R. J. Stephenson, M. E. Welland, C. Gerber, and J. K. Gimzewski, Photothermal Spectroscopy with Femtojoule Sensitivity Using a Micromechanical Device, Nature 372, 79 (1994).

[34] A. R. Krause, C. Van Neste, L. Senesac, T. Thundat, and E. Finot, Trace Explosive Detection Using Photothermal Deflection Spectroscopy, J. Appl. Phys. 103, 094906 (2008).

[35] S. Kim, D. Lee, and T. Thundat, Photothermal Cantilever Deflection Spectroscopy, EPJ Tech. Instrum. 1, 7 (2014).

[36] E. A. Wachter and T. Thundat, Micromechanical Sensors for Chemical and Physical Measurements, Rev. Sci. Instrum. 66, 3662 (1995).

[37] N. Umeda, Scanning Attractive Force Microscope Using Photothermal Vibration, J. Vac. Sci. Technol. B Microelectron. Nanom. Struct. 9, 1318 (1991).

[38] G. C. Ratcliff, D. A. Erie, and R. Superfine, Photothermal Modulation for Oscillating Mode Atomic Force Microscopy in Solution, Appl. Phys. Lett. 72, 1911 (1998).

[39] H. Yamashita, N. Kodera, A. Miyagi, T. Uchihashi, D. Yamamoto, and T. Ando, Tip-Sample Distance Control Using Photothermal Actuation of a Small Cantilever for High-Speed Atomic Force Microscopy, Rev. Sci. Instrum. 78, 083702 (2007).

[40] S. W. Stahl, E. M. Puchner, and H. E. Gaub, Photothermal Cantilever Actuation for Fast Single-Molecule Force Spectroscopy, Rev. Sci. Instrum. 80, 073702 (2009).

[41] D. Ramos, J. Tamayo, J. Mertens, and M. Calleja, Photothermal Excitation of Microcantilevers in Liquids, J. Appl. Phys. 99, 124904 (2006).

[42] S. Saraf, R. Shikler, J. Yang, and Y. Rosenwaks, Microscopic Surface Photovoltage Spectroscopy, Appl. Phys. Lett. 80, 2586 (2002).

[43] L. Malikova, T. Holden, M. N. Perez-Paz, M. Muñoz, and M. C. Tamargo, Microscopic Surface Photovoltage Spectroscopy of the CdSe/ZnCdMgSe Quantum Dots Structures, Appl. Phys. Lett. 94, 102109 (2009).

[44] Z. Schumacher, Y. Miyahara, A. Spielhofer, and P. Grutter, Measurement of Surface Photovoltage by Atomic Force Microscopy under Pulsed Illumination, Phys. Rev. Appl. 5, 044018 (2016).

[45] M. Gwon, A. Sohn, Y. Cho, S. H. Phark, J. Ko, Y. Sang Kim, and D. W. Kim, Plasmon-Enhanced Surface Pho- 
tovoltage of $\mathrm{ZnO} / \mathrm{Ag}$ Nanogratings, Sci. Rep. 5, 1 (2015).

[46] M. Alexe and D. Hesse, Tip-Enhanced Photovoltaic Effects in Bismuth Ferrite, Nat. Commun. 2, 1 (2011).

[47] Z. D. Luo, D. S. Park, M. M. Yang, and M. Alexe, LightControlled Nanoscopic Writing of Electronic Memories Using the Tip-Enhanced Bulk Photovoltaic Effect, ACS Appl. Mater. Interfaces 11, 8276 (2019).

[48] M. V. Rastei, F. Gellé, G. Schmerber, A. Quattropani, T. Fix, A. Dinia, A. Slaoui, and S. Colis, Thickness Dependence and Strain Effects in Ferroelectric Bi2FeCrO6 Thin Films, ACS Appl. Energy Mater. 2, 8550 (2019).

[49] J. Jahng, J. Brocious, D. A. Fishman, F. Huang, X. Li, V. A. Tamma, H. K. Wickramasinghe, and E. O. Potma, Gradient and Scattering Forces in Photoinduced Force Microscopy, Phys. Rev. B, 90, 155417 (2014).

[50] D. Nowak, W. Morrison, H. K. Wickramasinghe, J. Jahng, E. Potma, L. Wan, R. Ruiz, T. R. Albrecht, K. Schmidt, J. Frommer, D. P. Sanders, and S. Park, Nanoscale Chemical Imaging by Photoinduced Force Microscopy, Sci. Adv. 2, e1501571 (2016).

[51] T. Tumkur, X. Yang, C. Zhang, J. Yang, Y. Zhang, G. V. Naik, P. Nordlander, and N. J. Halas, WavelengthDependent Optical Force Imaging of Bimetallic Al-Au Heterodimers, Nano Lett. 18, 2040 (2018).

[52] R. Meyer, S. Trautmann, K. Rezaei, A. George, A. Turchanin, and V. Deckert, Synergy of Photoinduced Force Microscopy and Tip-Enhanced Raman Spectroscopy - A Correlative Study on MoS 2, ACS Photonics 6, 1191 (2019).

[53] A. Ambrosio, R. C. Devlin, F. Capasso, and W. L. Wilson, Observation of Nanoscale Refractive Index Contrast via Photoinduced Force Microscopy, ACS Photonics 4, 846 (2017).

[54] F. Huang, V. A. Tamma, Z. Mardy, J. Burdett, and H. K. Wickramasinghe, Imaging Nanoscale Electromagnetic Near-Field Distributions Using Optical Forces, Sci. Rep. 5, 1 (2015).

[55] J. Jahng, D. A. Fishman, S. Park, D. B. Nowak, W. A. Morrison, H. K. Wickramasinghe, and E. O. Potma, Linear and Nonlinear Optical Spectroscopy at the Nanoscale with Photoinduced Force Microscopy, Acc. Chem. Res. 48, 2671 (2015).

[56] T. U. Tumkur, M. A. Hurier, M. D. Pichois, M. Vomir, B. Donnio, J. L. Gallani, and M. V. Rastei, Photoinduced Atomic Force Spectroscopy and Imaging of TwoDimensional Materials, Phys. Rev. Appl. 11, 044066 (2019).

[57] B. Kim and E. O. Potma, Laser Heating of Cantilevered Tips: Implications for Photoinduced Force Microscopy,
Phys. Rev. B 100, 195416 (2019).

[58] J. Yamanishi, Y. Naitoh, Y. J. Li, and Y. Sugawara, Heterodyne Technique in Photoinduced Force Microscopy with Photothermal Effect, Appl. Phys. Lett. 110, 123102 (2017).

[59] L. Wang, D. S. Jakob, H. Wang, A. Apostolos, M. M. Pires, and X. G. Xu, Generalized Heterodyne Configurations for Photoinduced Force Microscopy, Anal. Chem. 91, 13251 (2019).

[60] Y. Liu, S. Begin-Colin, B. P. Pichon, C. Leuvrey, D. Ihiawakrim, M. Rastei, G. Schmerber, M. Vomir, and J. Y. Bigot, Two Dimensional Dipolar Coupling in Monolayers of Silver and Gold Nanoparticles on a Dielectric Substrate, Nanoscale 6, 12080 (2014).

[61] O. Kovalenko, M. Vomir, B. Donnio, J. L. Gallani, and M. V. Rastei, Chiromagnetoptics of Au and Ag Nanoparticulate Systems, J. Phys. Chem. C, 124, 21722, (2020).

[62] M. Guerrisi, R. Rosei, P. Winsemius, Splitting of the interband absorption edge in Au. Phys. Rev. B, 121975, 557 (1975).

[63] A. V. Pinon, M. Wierez-Kien, A. D. Craciun, N. Beyer, J. L. Gallani, and M. V. Rastei, Thermal Effects on van der Waals Adhesive Forces, Phys. Rev. B 93, 035424 (2016).

[64] M. Wierez-Kien, A. D. Craciun, A. V Pinon, S. Le Roux, J. L. Gallani, and M. V Rastei, Interface Bonding in Silicon Oxide Nanocontacts: Interaction Potentials and Force Measurements, Nanotechnology 29, 155704 (2018).

[65] Data for the full displacement interval is shown in the Supplemental Material.

[66] D. Ramos, J. Mertens, M. Calleja, and J. Tamayo, Photothermal self-excitation of nanomechanical resonators in liquids, Appl. Phys. Lett. 92, 173108 (2008).

[67] A. Barsella, M. A. Hurier, M. D. Pichois, M. Vomir, H. Hasan, L. Mager, B. Donnio, J. L. Gallani, and M. V. Rastei, Photonic excitation of a micromechanical cantilever in electrostatic fields, Phys. Rev. Lett. 125, 254301 (2020).

[68] D. Ma, J. L. Garrett, and J. N. Munday, Quantitative measurement of radiation pressure on a microcantilever in ambient environment, Appl. Phys. Lett. 106, 091107 (2015).

[69] M. H. Chien, M. Brameshuber, B. K. Rossboth, G. J. Schütz, and S. Schmid, Single-molecule optical absorption imaging by nanomechanical photothermal sensing, PNAS, 115, 11150 (2018).

[70] D. Ramos, O. Malvar, Z. J. Davis, J. Tamayo, and M. Calleja, Nanomechanical Plasmon Spectroscopy of Single Gold Nanoparticles, Nano Lett. 7165, 18 (2018).

[71] Garcia, R. and Perez, R. Dynamic atomic force microscopy methods, Surf. Sci. Rep. 47, 197-301 (2002). 